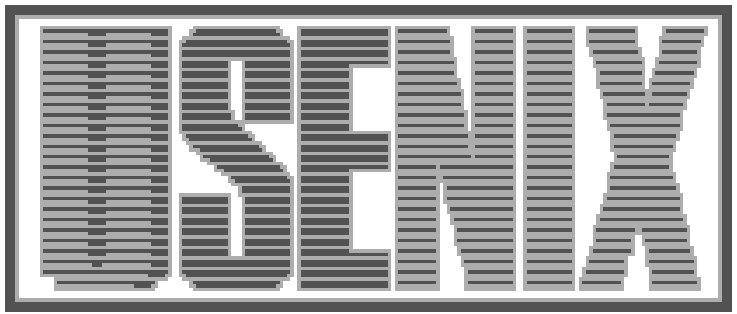

The following paper was originally published in the

Proceedings of the 4th USENIX Conference on Object-Oriented Technologies and Systems (COOTS)

Santa Fe, New Mexico, April 27-30, 1998

\title{
Mobile Objects and Agents (MOA)
}

Dejan S. Milojicicc, William LaForge, and Deepika Chauhan

The Open Group Research Institute

For more information about USENIX Association contact:
1. Phone:
$510528-8649$
2. FAX:
$510548-5738$
3. Email:
office@usenix.org
4. WWW URL: http://www.usenix.org/ 


\title{
Mobile Objects and Agents (MOA)
}

\author{
Dejan S. Milojičić, William LaForge, and Deepika Chauhan \\ The Open Group Research Institute \\ [dejan, laforge, dchauhan]@opengroup.org
}

\begin{abstract}
This paper describes the design and implementation of the Mobile Objects and Agents (MOA) project at the Open Group Research Institute. MOA was designed to support migration, communication and control of agents. It was implemented on top of the Java Virtual Machine, without any modifications to it. The initial project goals were to support communication across agent migration, as a means for collaborative work; and to provide extensive resource control, as a basic support for countering denial of service attacks. In the course of the project we added two further goals: compliance with the Java Beans component model which provides for additional configurability and customization of agent system and agent applications; and interoperability which allows cooperation with other agent systems.

This paper analyzes the architecture of MOA, in particular the support for mobility, naming and locating, communication, and resource management. Object and component models of MOA are discussed and some implementation details described. We summarize the lessons learned while developing and implementing MOA and compare it to related work.
\end{abstract}

\section{Introduction}

Mobility has always attracted researchers in computer science. This interest spans from general observations, such as "if it weren't for mobility, we would still be trees" [10], and the analogies with the real world "migrating birds and nomadic tribes moving due to the lack of resources", to purely technical reasons, such as improving locality of reference and difference between local and remote semantics.

One of the first incarnations of software mobile entities. is worms [30], which could spread across nodes and arbitrarily clone. Unrestricted implementations of worms and viruses have received negative connotations, due to security breaches and denial of service attacks [13].

The next generation of mobile entities, known as process migration, were implemented at the operating sys-

This work was supported in part by the Advanced Research Projects Agency and the Rome Laboratory of the Air Force Materiel Command. tem (OS) level. There were many implementations of process and object migration $[3,12,19,31]$, but none has achieved wide acceptance. Due to inherent complexity, it was hard to introduce process migration without impacting the stability and robustness of the underlying OS.

Mobile objects and agents have attracted significant attention recently. In addition to mobile code (such as applets), agents consist of data and non-transient system state that can travel between the nodes in a distributed system (intranet or Internet). Compared to mobile objects, mobile agents also represent someone; they can perform autonomous actions on behalf of a user or another agent. A number of academic systems (such as Agent Tcl [20], Mole [4], Ara [27] and Tacoma [18]) and industrial systems (such as Telescript [34], Aglets [1], Concordia [9] and Voyager [33]) exist. The products using mobile agents have started to appear, such as Guideware [16]. The government is interested in funding work on agents [11]. A patent has been approved on mobile agents [35]. A standard has been adopted (OMG MASIF [26]), and reference implementations are in progress. A couple of books have been published on agents $[6,8]$ and a few more are in progress [21, 24].

This paper describes the Mobile Objects and Agents (MOA) project at the Open Group Research Institute. The obvious question is why yet another mobile agent system? There were a few reasons. None of the existing systems at the time of starting the project were mature enough to be used as a starting point for our work. We found it easier to develop another system that would suit our needs from the beginning. Additionally, some areas of our interest, such as communication and resource control, are deeply involved in the design decisions of any system, making it very hard to add them as an afterthought. Finally, we were interested in interoperability between the systems, and therefore supporting another implementation was a good idea.

At the beginning of the project we were interested in the first two of the four features listed below, and during the course of development we added the last two:

Collaboration. Frequently, agents need to collaborate during their execution either with other agents or their 
user. For agent collaboration, it is required to support naming, locating and communication among agents.

Denial of service attacks. Agents, as well as hosts, are vulnerable to mutual attacks, either over a network or locally. In order to prevent denial of service attacks, it is required to maintain resource control of agents and agent systems, and to impose security and resource policies.

Configurability and customization. It is increasingly difficult to configure and customize software. In the case of mobile agents, this applies both to agent applications, as well as to agent systems. Being compliant with a component model, such as Java Beans, allows for a standardized way to access and change component properties.

Interoperability. Agents, as well as agent systems, need to interoperate. In the case of agent systems, interoperability leads to a larger base that agents can visit. We were active in the OMG Mobile Agent Facility proposal which addresses mobile agents systems interoperability [26].

More details on how these goals have been achieved is described in Sections 4.3, 4.6, 4.2 and 4.10 respectively.

The rest of this paper is organized as follows. In Section 2 we provide a background on mobile agents and component-based computing. Section 3 describes Java's suitability for mobile agents and for component-based computing. Section 4 presents the MOA design and implementation. Section 5 discusses MOA current status. Section 6 describes some MOA applications. In Section 7 we present lessons learned while designing and implementing MOA. MOA is compared to related work in Section 8. Finally conclusions and future work are presented in Section 9.

\section{Background}

In this section, we provide background on mobile agents and component-based computing.

\subsection{Mobile Agents}

Among the benefits of mobile agents we would like to underline the following. Improving locality of reference is achieved by moving the action towards the source of data or other end point of communication, resulting in substantial performance improvement. Survivability: similar to nomadic tribes or migratory birds, agents can survive if moved closer to resources, or away from partially failed nodes. Analogy to the real world helps some programmers to better understand programming paradigms expressed in terms of mobile agents. Examples are travelling salesman, shoppers and workflow management systems. Customization of software can be achieved using mobile agents, for example, by adjusting the search according to a user-specific criteria, or by per- forming an action specific to a remote site. Autonomicity represents agent's independence from its owner. A user can start an agent to act on his behalf and disconnect. When the user reconnects, the agent returns or otherwise provides results.

Agents have various areas of deployment. One is slow and unreliable links, such as radio communication, where locality of reference improves performance, and avoids potential loss while transferring large amounts of data. Software distribution becomes increasingly hard. Mobile code has provided a revolutionary breakthrough, by allowing downloading code for heterogeneous environment. Mobile agents makes this effort even easier, by associating actions and state with each distributed version and copy of a particular software. Network management: agents migrate both code and data, making them useful for automating control and configuration in large scale environments, such as networks [15]. Electronic commerce deploys mobile agents by modeling travelling salesmen or shoppers visiting stores in an electronic mall. Data mining is a convenient application for mobile agents due to locality of reference: agents optimize a search by wandering from site to site with large volumes of information. (See [7] for additional benefits.)

Nevertheless, mobile agents still haven't achieved wide acceptance. Some of the reasons include the following. Lack of applications: mobile agents have achieved a reputation of "the solution searching for the problem". Many systems have been developed but few applications exist. Security: the problems caused by mobile code are frequently reported. Mobile agents push the security problems even further. Lack of infrastructure adapted for mobile agents, such as name servers, messaging systems, and management, is still not widely deployed. Survivability is both a benefit and a challenge for mobile agents. Mobile agents are inherently survivable, but this does not come free; they need to be designed and implemented for survivability. In particular, they should minimize residual dependencies on previously visited nodes, or servers.

\subsection{Component-Based Computing}

Component-based programming, including OpenDoc, VBX, and ActiveX, has been quite successful in speeding the development of GUI applications. Java Beans (components written in Java), are promising for nonGUI component programming. The runtime behavior of a Java Bean is defined by an ordinary Java class. The difference between a bean and other objects is the metadata used for configuration. It is provided by an associated BeanInfo class, or it is derived from the runtime class. 
Component-based programming enhances object oriented benefits, such as flexibility, and code reuse with two new characteristics: independence and configuration.

Independence. The source code defining a component does not directly reference any other component; instead, relationships between components are created at runtime. The relationships may be established by the container holding the components, or even by the component itself. This has several benefits:

- a "building block" approach: programs are constructed from existing components by defining relationships

- each component can be individually tested

- components are more easily reused; there is a minimum of interdependency between components

- a program can be restructured for new requirements without impacting the logic of individual components

- updating a program with the latest version of 3rd party components is simplified

Configuration. A component is constructed by a general configuration tool. The component participates in its own configuration. Application programs are assembled from pre-configured components. The implementation specifics of a component are separated from other elements of the program. Separating the configuration of components from an application program facilitates the use of alternative implementations and component upgrades are backward compatible. However, this impacts the development cycle, as changes made to a component's source code will often invalidate its configuration. The edit, compile, and test of the development cycle now becomes edit, compile, configure, and test.

\section{Java}

We have chosen Java because it seemed to be the mainstream programming language, but also because of the features that make it suitable for mobile agents and components.

\subsection{Java and Mobile Agents}

Java offers advantages for mobile agents, as well as some disadvantages. Advantages consist of the support for mobile code, heterogeneity, language safety, object serialization [28], reflection, dynamic class loading, and multithreading.

Disadvantages consist of inadequate support for resource management (e.g. memory and disk limits), no support for preserving the thread execution context, limited support for versioning, no ownership of objects and fine grained protection at the object granularity [21].

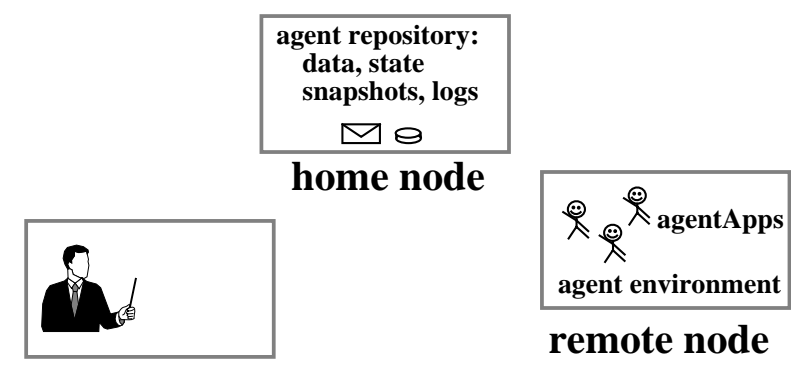

front-end node

Figure 1. MOA Configurations: front-end supports starting and controlling agents and other MOA components. Home node is the node where agent was originally started and where agent-related state is maintained. Remote node is one of the nodes where agent currently executes.

\subsection{Java and Components}

Components written in Java are lightweight and little code is required for conformance to the component model. Java supports a number of key features of component programming:

- A component may have several interfaces. Java provides for the implementation of multiple interfaces, unrestricted casting, and an instanceof operator to determine if a component supports an interface.

- Several components may be aggregated into a single component. The JDK 1.1 methods Beans.isInstanceOf and Beans.getInstanceOf can be used in place of the instanceof operator and casting, allowing for the future use of aggregation in JDK 1.2.

- The life of a component may span more than one program. JDK's provision for serializing components allows converting an object into a form which can be written to a disk file or passed across a network.

- A component is configured by modifying its properties identified by examining the method signatures of the component, e.g. the class is recognized as having the property slices, if the methods getSlices and setSlices exist.

- The component's properties are accessed using the class Introspector.

\section{MOA Design and Implementation}

The MOA architecture is presented in Figure 1. There are three types of nodes that run MOA system: front-end node allows users to control and monitor agents; home node is used as a repository for agent's data; and remote node is where agents typically run throughout their life time. The MOA system has a Telescript-like model (although sufficient difference avoids infringing their patent). Agents travel visiting places held by Agent Environments (AE). Places accept agents, and store information. Agent environments host various objects. A 
name server tracks the location of agents and other objects, whereas a monitor serves for controlling and monitoring objects. These and other objects in the MOA architecture are described in more detail in this section.

\subsection{Object Model}

The MOA objects on remote nodes can be classified as agent- or system-related (see Figure 2). Agent-related objects are circled; they have migratory state. Agent and place belong to the user trust domain (see Section 4.7 on more details related to security), whereas other components belong to the MOA trust domain.

An agent and place are application extended classes. Agent is the first class MOA object. It is a template class extended by agent applications. Agents are named (see Section 4.4), and they can communicate (see Section 4.3). An agent can move to an agent environment (or a place within it), it can request to meet other agents at a certain place or agent environment, openChannel to another agent, or sendMessages to it. An agent always executes within a place (see below). There is a one-toone mapping between an agent and a place within an agent system. However, an agent can leave places behind when it moves. Therefore there is one-to-many mapping between an agent and places on different agent systems.

Place is the second class MOA object. The main difference from agent is that place is a stationary object, and therefore it can not move or meet. However, places can communicate with other places and agents; they can be active, i.e. they can have threads running. Place also serves the container-proxy role. They are proxies because they can remain after an agent leaves and represent it there (be proxy). They serve the container role for security and resources of an agent.

Agent Control, Agent Properties, Family, LogService and Bucket are agent-related classes that belong to the MOA trust domain. Agent control is an internal class that represents an interface between agent system and agent/place. It manages agent system resources (communication channels, agent properties, etc.). These objects can be accessed by an agent/place, but they cannot be changed. AgentProperties contains the properties that characterize agents and are transferred across the nodes. Examples are owner, home Agent Environment, and locatingStrategy. Family is used for monitoring agent activities. Each agent has its own Family object which it carries across migrations. LogService manages local logs. Bucket holds the contents of a JAR file (JAva aRchive). Each bucket implements a classloader for the dynamic loading of the jar file. A hashtable contained within a bucket enables the client of a bucket to efficiently index into the contents of a jar file. During migration,

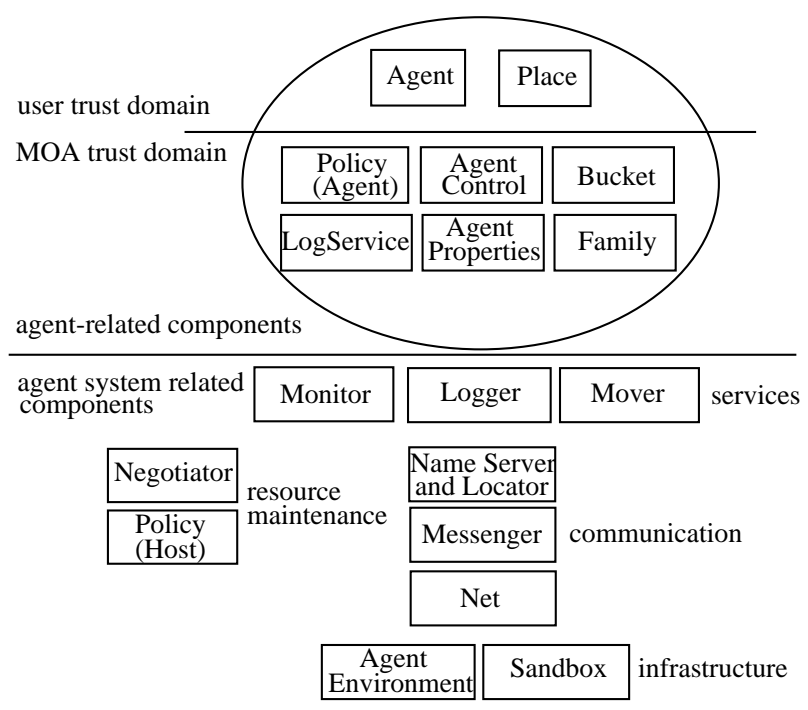

Figure 2. MOA Objects: consist of objects in user trust domain, and MOA objects in MOA trust domain. Agent environment represents container for all MOA objects.

components circled in Figure 2 (except for Place and Agent Control) are serialized, put into the bucket and sent to destination node.

Policy and Negotiator objects maintain and manage information about resources. Policy is a placeholder for properties describing the policy of an agent arriving at a node (agentPolicy), and a host receiving the agent (hostPolicy), such as agent's maximum lifetime, maximum number of channels and maximum threads. Negotiator performs negotiation between the agent and the receiving agent environment prior to agent's visit. Agent movement is subject to resource requirements and security arrangements between the two entities.

Sandbox and Agent Environment provide basic infrastructure. Sandbox class separates the agent application from the agent system state. It switches from the agent system thread to application thread when there is a different protection domain; it also serves to switch from synchronous to asynchronous communication when going across the network. Resource usage and limits are tracked on a per sandbox basis. AgentEnvironment $(\mathrm{AE})$ is the container for agents and their related objects at an agent system. There is one agent environment per Java Virtual Machine (JVM), but there can be many per a node. Each agent has its home AE and alternate home $\mathrm{AE}$ in case the home is not accessible.

Net, Messenger and Name server comprise the MOA communication model. Net provides the basic communication support for establishing and maintaining communication channels between components on remote and local JVMs. Communication channels are established by specifying the component name, host and port number. 


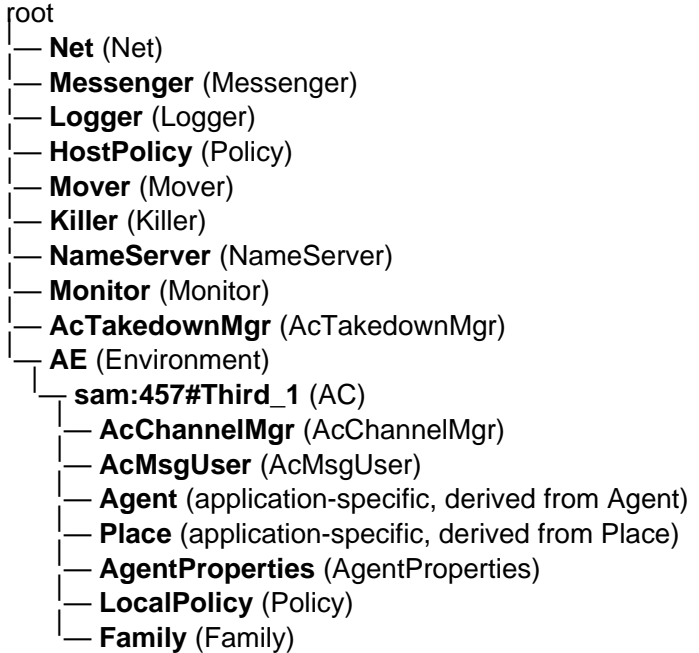

Figure 3. Agent System Object Tree defines the names of MOA internal objects. Parenthesis contain the class names from which the objects are derived. Internal object names are important when communication between various objects on different MOA systems is established, and for initialization.

The Messenger layer uses the services of Net to support one- and two-way messages between components. Components are addressed using destination agent system and the component name. Name server tracks agent locations. The name server clients can (un)register and lookup an agent location. Name server clients are user (monitor) and agents that lookup locations of other agents in order to communicate. Information about the agent location (or how to find it) is cached at agent systems that the agent visited or communicated with. Name server also plays the Locator role.

Mover, Monitor and Logger provide MOA services. Mover supports agent movement. It negotiates migration, captures the agent state, and transfers it. Monitor provides a user interface to control and monitor applications (e.g. agent's movement, communication and resource usage). Logger logs events in an Agent System to persistent media.

MoaApplet, BatchDriver and User classes support the interface to the MOA system and its applications. MoaApplet is an applet-based interface enabling users to interactively monitor and debug agents, to launch them, to snapshot the agent's state, and to query the logged data. BatchDriver is a script-based user interface to provide the services of MoaApplet; typically it is used for testing purposes. User class serves as an interface between the user applet and the agent. It launches the preconfigured agents, tracks the agents, and maintains information of interest to a user.

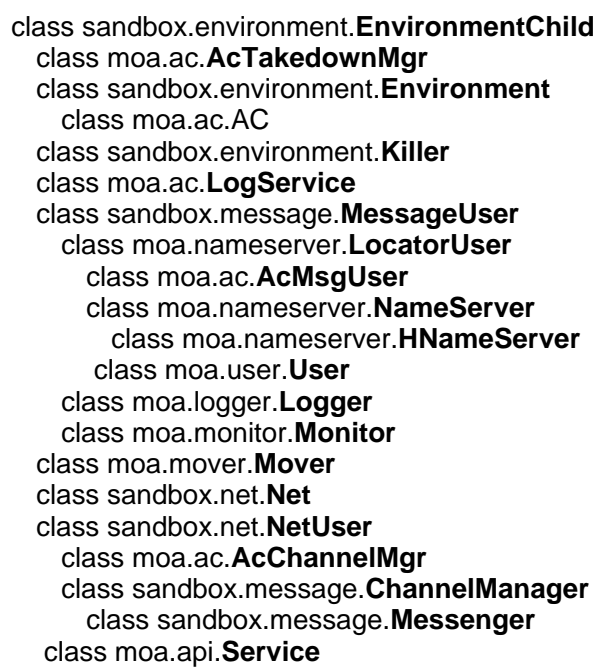

Figure 4. Inheritance tree of EnvironmentChild Class: the EnvironmentChild class supports accessing other components within the object tree (see Figure 3). For example, the Mover needs access to NameServer and Messenger and therefore has to be wired. Inheritance tree indicates objects accessible by inheritance, for example, Mover has access to Messenger by being a subclass of MessageUser, and needs not be wired.

\subsection{Component Model}

MOA components are configured using the MOAbatch tool (see Section 5). The components configuration defines the system object tree. The MOA system is loaded by first loading the root component. Each component then successively loads the component below it in the tree. Components are locally organized into a labeled tree (see Figure 3) used to dynamically establish relationship between the components, in contrast to static binding, typical of $\mathrm{OO}$ programming.

After the components have been loaded, they can locate other components of the agent system by name in order to establish dynamic binding. Non-leaf elements of the object tree subclass the environmentChild class (see Figure 4) which provides methods for locating a tree element given a relative or full pathname. For example, any component in Figure 4 can access the Mover object by calling the method:

getEnvironment().getInstanceOf(“/Mover”,Mover.class)

Coupling of components to a certain extent negates the benefits of component programming, and as such it has been kept to a minimum. For example, the net component is made known to other components, such as those that subclass NetUser: AC, Messenger and arbitrary applications using Net.

We have successfully used components for preconfiguring agent applications, as well as the agent system. For agent applications, we can easily preconfigure an agent's itinerary, policy, types of logging, debugging, etc. Agent system configuration specifies which components will 
Debug (List of components being debugged)

AE (agent application components)

Messenger (netBean,timeout)

Monitor (messengerBean,timeout)

Mover (messengerBean,timeout)

Net (retryDelays(Increment,max,init),runSrv, host,port,exitSrvOnError)

HomeService (nameSrvBean, messengerBean)

Logger (fileSuffix,logPath, retentionPeriod,messengerBean,timeOut)

NameServer (nameSrvBean, messengerBean)

Users (userPath, nameSrvBean,passwd,messengerBean,HSretryDelay)

UE (listOfUserBeans)

HostPolicy (maxLifeTime,timeRemain, maxChnl,remainPlaces, maxThrd)

Killer (killerTimeout)

Root (AE,debugger,net, messenger,logger, hostPolicy, mover, nameSrv UE, homeServer, monitor,ACTakeDownMgr,killer)

ACChannelManager (timeout, netBean)

AcMsgUser (nameSrvBean, messengerBean,timeout)

AC (AcChannelMgr,AcMsgUser)

Figure 5. MOA Components, described with the list of properties (configuration of the remote MOA system).

be integrated into the tree. For example, homeService is not present on all agent systems, and agent environment is not needed on the front-end and on the home node. Debugging can be specified as a part of the agent system configuration at class granularity. Message timeouts can be configured on a component basis. For the Net component we specify its port number; for each agent, we specify the number of service threads for each agent; Killer component's property includes time when it will take the system down; for each user, we specify the password, login id and login time; for each system, we specify the host policy for negotiating with agents.

Components can also be organized using subcomponents. For example, AC is an aggregate which includes AcChannelMgr and AcMsgUser. Only AC is configured into the whole system. This way configuration is simplified using a hierarchical structure. The MOA components of a remote system and their properties are presented in Figure 5.

\subsection{Communication}

The MOA communication is built on top of JVM sockets. It provides a higher level of abstraction, such as the channels and messaging between MOA objects (agents, places and servers). The communication channels support object streams. Messaging provides for passing objects of arbitrary type specific to the application.

The Net package supports opening of channels with automated retry. The destination system can optionally reject a request for the channel subject to resource limitations. This can happen at the agent system, as well as at the application level. When opening a channel to an agent, only the agent name needs to be specified. The agent system resolves the actual agent location with the help of Locator object in a distributed manner.

The Message package is able to pass application specific objects by delaying deserialization until the name space of destination is identified. Messages can be synchronous (RPC-like), or asynchronous (one-way messages).

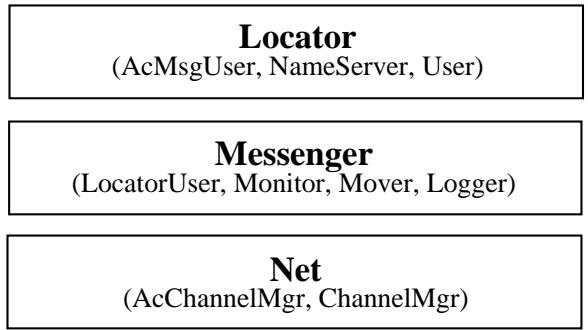

Figure 6. Stacked Communications Layers: Net supports stream based communication, messenger messaging and locator introduces transparent locating of migratory objects.

Messaging is built on top of the Net package, using a common pool of channels dedicated to message passing. The destination of messages can either be a specific location (destination name, host and port), or a logical name (e.g. agent name) in the case where the destination is a migrateable object. This is reflected in the implementation, where a layered approach is applied by building the Locator on top of the Message layer which builds on top of the Net (see Figure 6). The Locator handles transparent routing of messages when a location is not specified. It enables the agents to transparently communicate and collaborate with each other by using the name of the agent. The Locator relies on the locating strategies described in Section 4.4 to find new agent location.

In the case of two way messages, responses are routed back to the originating thread, which is suspended pending either a response or a time out. A response can arrive from the node other than the original destination, if the destination agent moved.

While moving from one node to another, the agent does not notice that its channels have been closed and reopened on the remote node. When the transfer is initiated, the channel migration process is performed first. During this process, the agent informs its collaborating partners of its intent. From this point onwards, the data received on the channels is not passed to the application, but is stored in a Vector of unread Objects. Upon learning about the move, the agent channel manager on the other end of the channel replies back an acknowledgment and closes the channel socket without informing the application. For the migrating agent, when the acknowledgment is received on a channel, the Reader thread for the channel is stopped, and the socket is closed. The agent transports itself to a new location, only when the migration process is completed for all open channels.

During channel migration, though the socket is closed, the information regarding the other communicating agents/places is still maintained. When the agent moves, it carries along the information about the channels, and uses it to reopen channels at the new location. Prior to reopening channels it first sends all the unread objects to 


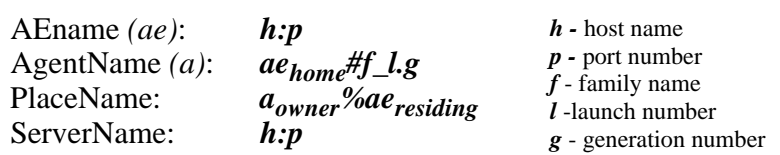

Figure 7. MOA Named Objects: Agent Environment, Agent, Place and Server

the application. Reopening of channels can be done either eagerly or lazily, depending on the type of the application. If there are many channels not used often, they are re-opened lazily. If there are a few channels likely to be used after migration, then they are reopened eagerly.

MOA uses remote method invocation within a number of its components. It would have been a reasonable choice to rely on Javasoft's RMI [36] instead, but we have not made such a decision. There is nothing to prevent us from using it in the future (and most likely we shall switch to using it), but for the initial implementation we did not want to adopt yet another technology (in addition to Java Beans) that would introduce a learning curve. Furthermore the unclear situation with CORBA v. RMI was another contributing factor. Instead of using RMI, we simulate its functionality by sending objects across the network; based on the object type we invoke appropriate methods. The actual implementation is trivial. There are a small number of uses of remote method invocation and these are confined to limited scope of the MOA implementation, whereas MOA applications can use RMI. Overall, it was more a political- rather than a technical-decision not to use RMI for the initial implementation.

Communication and resource management are deeply involved in the design decisions in MOA. This is reflected in many MOA layers and components. For example, communication is involved in the communication stack (Net, Message, NameServer and Locator), but also in the sandbox, AC, and agent/place interfaces. Similar applies to resource management. The AC and sandbox components were shaped to enable resource tracking. It would have been hard to add this support as an afterthought. Our earlier experience with Mach task migration [23] indicates that to a certain extent it is possible to add resource management or to make communication modifications as an afterthought, however, any significant support needs to be well elaborated in advance.

\subsection{Naming and Locating}

The following objects are named in a MOA system: agent environment, agent, place and servers. Name syntax is presented in Figure 7. An agent environment is named after the node and port on which they perform communication. A server name has similar syntax.

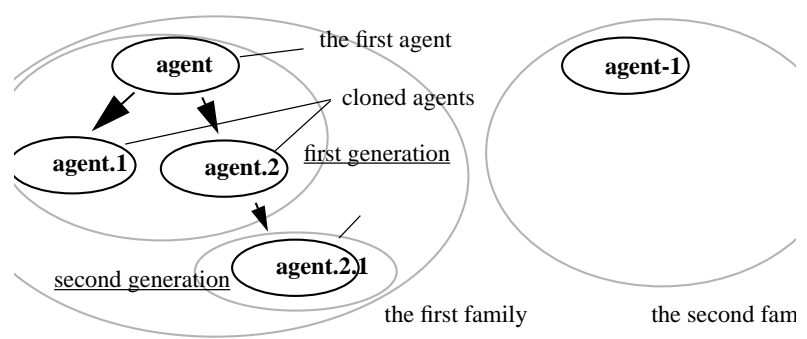

Figure 8. Naming of agents: agent names are organized around agent families and generations. Cloned agents always carry the name of its ancestor as a part of their name.

Agents are named after the AE where they were created if they are first generation. If cloned, agents are named after the AE of their first ancestor extended with the generation number (see Figure 8), irrespective of the $\mathrm{AE}$ where they were actually cloned. In other words, each agent bears the sign of the original site responsible for initiating the agent family. This is used as an ultimate source of information on the current agent location: as a last resort, the home AE can be queried for current location information. The place name consists of the name of an agent it belongs to, extended with the AE name where place currently resides.

The agent location may be needed by its owner explicitly in order to track the agent location, or implicitly in order to be able to control it (kill, suspend, resume, etc.). It is also needed by agents in order to be able to communicate to (open channels) or synchronize (propose meeting) with other agents.

Locating agents is performed through name servers. Application requests to name servers (lookup, register, unregister) are issued through the agent environment which performs security and consistency verifications. When migrating the agent, the Mover object makes a local request to the Name Server. When opening channels or sending messages, NetUser and Messenger interact with the Name Server. Name servers on multiple nodes then cooperate to satisfy these requests.

The location object contains either the current location of an agent, or sufficient information to obtain it. In particular, it contains some or all of the following: the name of the residing agent system (if known), the type of the strategy to locate the agent (discussed below), the list of the nodes where the agent may reside (itinerary), the lifetime of this location object. Even though the lifetime of an agent is limited by its owner, because of the delays in transferring the agent over network, it is not possible to assure its accuracy.

The location object is cached at each node the agent visited, or where there was a channel opened with the agent. When searching for an agent, the location object is first looked up at a local name server. If not found, it is looked 
up at name servers higher in the hierarchy, if any exists (name servers may be organized in a tree-like hierarchy). If the agent is still not found, then the agent's home node is approached. The home agent environment is the ultimate source of information of agent location. The location of the home agent environment is implicitly known from the agent's name.

When locating agents, different location schemes are used, similarly to those used in distributed operating systems, such as Charlotte [3], V kernel [31], Sprite [12] and Mach [23]. The MOA system supports: a) updating the home after agent moves, b) registering at a predefined name server, c) searching based on predefined itinerary and d) forwarding based on the trails left after migration (see Figure 9). Locating scheme is selected subject to:

- destinations (a local or a far away region; limited set of destination hosts or unknown),

- security aspects (if agent crosses security domain)

- type of migration (burst v. sporadic; frequent v. rare; random v. cyclic).

For example, updating the home node is suitable for an agent that moves within a local region. It is not suitable for agents that visit distant nodes. Registering is suitable for an agent that migrates within a far away region; in the case of a large number of nodes, registering nodes are organized in a hierarchical manner; it is not suitable for a large number of migrations. Searching scheme is suitable for agents that visit a small number of known hosts; it is not suitable for destinations not known in advance and for large number of nodes visited. Forwarding is suitable for a small number of migrations; it is not appropriate for long chains.

In many cases, locating agents is application specific. Even if the agent is successfully located, it might migrate further away by the time the location is reported back to the requesting node and communication or delivery of a control message is attempted. This is especially critical in cases of control messages such as meet, suspend or kill. It is required to perform optimizations, such as to batch a locating request with the control message. This eliminates the delay between the time the agent is located and the control message is delivered to the visiting agent system. This may not be sufficient for highly dynamic agent applications or heavy loaded nodes in the case of forwarding locating strategy. Instead, the updating and/ or registering strategy needs to be used, combined with trapping the agent when registering/updating its location. This way agent movement is delayed until communication/control messages are delivered.

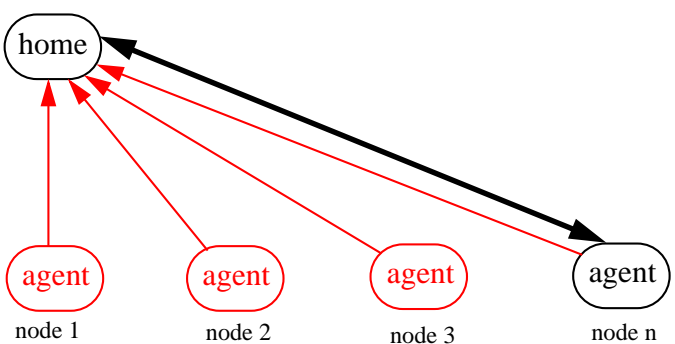

(a) updating at home node

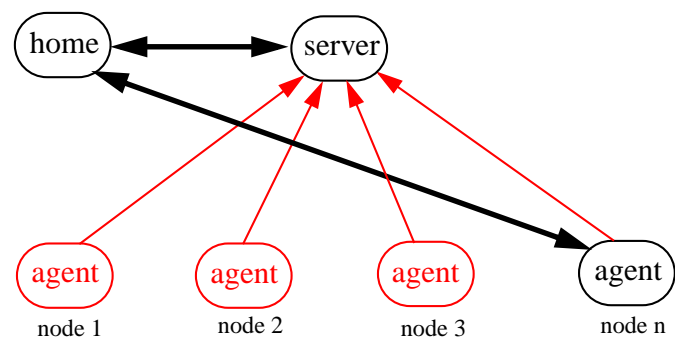

(b) registering

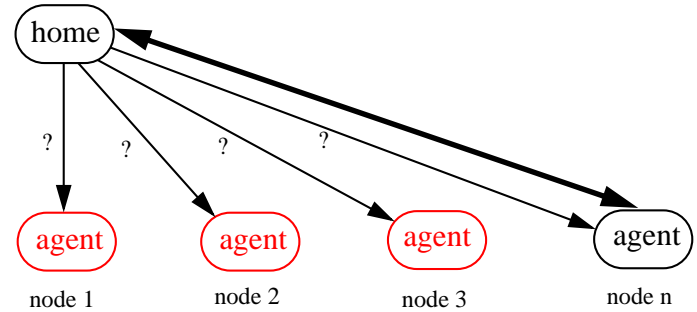

(c) searching

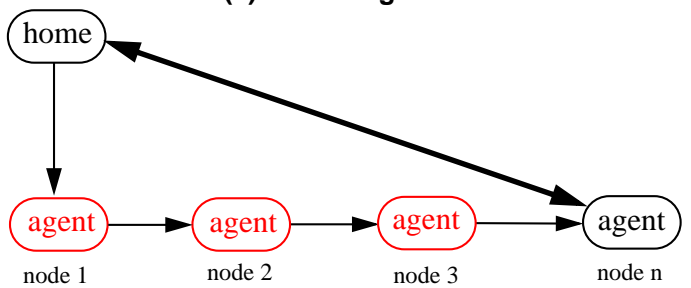

(d) forwarding

Figure 9. Locating schemes: (a) updating: an agent updates its location with the home node name server; $(b)$ registering: agent registers at a predefined name server; (c) searching: based on available itinerary, the sites are searched for agent's location; (d) forwarding based on trails agent left behind.

\subsection{Mobility}

When an agent migrates, its state is extracted from the source agent system and transferred to its destination where it is restored into a new instance of an agent object. During transfer, only site-independent information is transferred. In the case of communication channels, this information consists of the agent names with which the migrating agent had opened channels as well as their current location. The state relevant to each particular node is transient, i.e., it is discarded. For example the sockets maintained in the agent control object are closed 
AgentProperties (owner,familyName,home/alternateHomeAE,lifeTime) Agentpolicy (maxLifeTime,timeRemaining,maxChannel, remainingPlaces, maxThreads)

Family (typesOfLog,tracing,watches, limits)

Agent (applicationSpecific)

Place (applicationSpecific)

Figure 10. MOA agent applications Component Model: in parenthesis we present configurable properties.

and then reopened in the remote agent control object. Figure 10 describes the transferred agent state. The state extraction starts at the application level, where the application state is serialized (non-transient data), then the state of the agent control is serialized (agent resources, such as agent limits and logging data). This state is then transferred to the remote node through the cooperation of Mover objects in the source and destination agent system. The Mover objects involve negotiation based on the agentPolicy and the destination node hostPolicy.

Mobility is based on messaging, where the message object is the bucket containing the agent and related resources. When an agent arrives at a node, the Mover creates a new instance of the AC object (unless there already is one for that agent - the agent is returning to a place it left). All other agent-related objects are instantiated from the serialized versions in the bucket. Objects are loaded using the class loader associated with the bucket.

We do not provide for sharing of objects remotely, i.e. as an agent migrates to another node, it should not maintain any references to an object on the source node. Our experience is that distributed shared state is very hard to support at the system level [5], it is more appropriate to rely on distributed shared memory packages for such needs.

\subsection{Resource Management}

One of the initial goals of the MOA project was to support extensive resource control of various MOA resources. The following limits are enforced on MOA resources:

- agent: lifetime, places, hops, open channels, clones

- place: lifetime, nested places, open channels, agents

- agent environment: agents, places, channels

These limits are verified upon each MOA function that can impact the values, such as moving, or opening a channel. Should the limits be exceeded, the function is interrupted and the appropriate exception is thrown to the component that invoked the function.

Prior to being accepted at a node, the agent negotiates which and how many MOA resources it can utilize at the visiting MOA system. This is achieved by calculating local policy from the agent policy and host policy. The agent local policy is enforced during its lifetime at the visiting MOA system.
We did not address resource management not supported by JVM, such as the size of VM, the amount of processing, and communication. Whereas it would be possible to enforce some of them by making modifications to the JVM, we refrained from any deviation from de facto standard solutions. Imposing resource limits has impacted the design and implementation of the MOA system.

\subsection{Security}

The first MOA release is fully compatible with the JDK 1.1 security manager; however, no security manager has actually been implemented. Many security features were left open for the next release, such as the work on authentication, and authorization of agents. We have actually implemented only the following features.

Thread switching was employed to allow conformance with the Java security model. Services are provided by threads containing only trusted classes. When a MOA system thread has to switch the trust boundaries (for example in the case of an incoming message, or opening a channel), the request is passed to a system queue serviced by a pool of application threads allocated for that specific trust domain. A thread from the pool processes requests by calling the application specific methods. The request resumes either upon receipt of the response, or upon the timeout, whichever happens first. This way, the application is prevented from stalling the system by thread exhaustion, or by impacting performance through overusing system threads. In addition, resource usage is tracked on a per sandbox basis.

Each agent has its own name space as defined by the bucket in which it is transported. A name space consists primarily of bytecodes and serialized objects. One complication arises when an agent returns to a place that it had left. In this case, the name space is a combination of the original bytecodes and the returning objects. This is achieved by nesting the returning bucket (with the meaning of classloader in this context) within the bucket of the remaining place.

We are using the standard JAR file format for passing agents. This format has provision for digital signatures, allowing for authentication. However, we have not addressed authentication at the moment. It is left as an open issue, even though we have considered its deployment during design and implementation. For example, the agent's authenticity will be maintained as a part of the agent's name object.

\subsection{User Interface}

MOA's User Interface provides users with various types of interactive monitoring and debugging services. An applet-based and a script-based User Interface are provid- 
ed. The script-based interface is primarily used for testing purposes whereas the applet-based interface is used by the user to interact with the MOA system.

The User Interface is a component in the front-end of the system. It supports interaction with both the home and remote system. A user can log into the system from any remote location. The login information is verified at the home agent system and a list of all the agents (preconfigured applications available for launching and already launched applications) is returned to the user as a result of a successful login. The user can select any preconfigured application agent and launch it to a remote or local destination.

Users can launch a preconfigured agent, send a command to suspend or kill a selected agent and monitor agent-related activities. An agent can be queried by specifying its start time, duration (to determine which log records to access) and a query pattern. The home of an agent maintains a cyclic array of agent snapshots (captures of agent's state at different times). The user can fetch a snapshot and use it to start a new application agent. The interface accepts various types of messages pertaining to an agent's movement, notices, system statistics and log items. All these messages are displayable.

\subsection{MOA Tools}

We also developed the tools for manipulation of Java Beans. Even though many new tools are becoming available commercially, or will be developed soon, we needed some functionality that was not available at the time of development. In particular we developed MoaBatch program for instantiating Beans and MOAJar for manipulating JAR files.

MoaBatch is a simple script program (726 lines of code) which lets you instantiate beans (saving them to disk as serialized objects) and edit the properties. It can not use the property editors which do not support text. MoaBatch works with all of the property editors provided with the BDK except for the font editor. MoaBatch fully supports indexed properties. Source and executables are available from http://www.camb.opengroup.org/ laforge/java/moabatch/. Some of the commands that MoaBatch supports for manipulation of beans are included below:

- Instantiate X - create a bean X.ser.

- Properties X - list the properties of bean X.

- Limit N - limit display of elements of an indexed property

- Set X Y Z - set property Y of bean X to Z.

- SetAt X Y I Z - set property Y at I of bean X to Z.

MOAJar is a GUI utility for editing JAR files layered on top of an API for manipulating the JAR file contents and manifest. MoaJar supports:
- Add, remove, extract, or rename a file in the JAR.

- Edit the name/value attributes in the JAR manifest.

- Serialize object from a class in JAR or on CLASSPATH.

- Edit the properties of a serialized object in the JAR.

\subsection{Interoperability}

During the development of MOA, we participated in the OMG Mobile Agent Systems Interoperability Facility (MASIF) standard [26]. MASIF is an attempt by General Magic, IBM, Crystaliz, GMD and the Open Group to establish a standard for mobile agents using CORBA. It standardizes agent control, locating, and migration. It does not address communication among the agents. This participation was intertwined with the development of MOA. For example, our experience with MOA has impacted some of the choices in MASIF, and conversely, some of the MASIF specification choices have impacted MOA. In particular, our experience with locating contributed to standardization. MASIF impacted our selection of interfaces for the name server, as well as for the naming in future versions of MOA. OMG MASIF is important for enlarging the base of agent systems that can accept visiting agents.

\section{MOA Current Status and Performance}

MOA has been delivered to SECOM, utilizing funding provided by MITI. The project started in the summer of 1996. On average the project had four people working full time. Approximately 6 staff years were invested in the effort. The system is at the advanced research prototype stage. It has been tested for a number of scenarios, and we are currently conducting robustness and performance tests. MOA has been adopted as a base technology for a follow-up project ANIMA [2] in The Open Group Grenoble Research Institute. Three other sites have been using MOA: SECOM, University of Denver (further development of the Rent-a-Soft application), and INRIA (for security work).

MOA was developed on Windows NT, PC-based machines. We were mainly using the bare JDK for development, although throughout various phases of the project and for various purposes, developers have also been using other tools, such as Symantec Cafe, J++ and Java Studio. The main reason for using JDK was due to the relatively slow response of the industry to Java Beans development.

For development, we have used ATRIA's ClearCase. While we highly regard this tool in general and especially on UNIX machines (we had other development on HP/ UX), the match between Clearcase and the JDK on NT was not a good experience. The problems consisted of the use of upper case letters for file names, very poor response time and interference with debugging. By the 


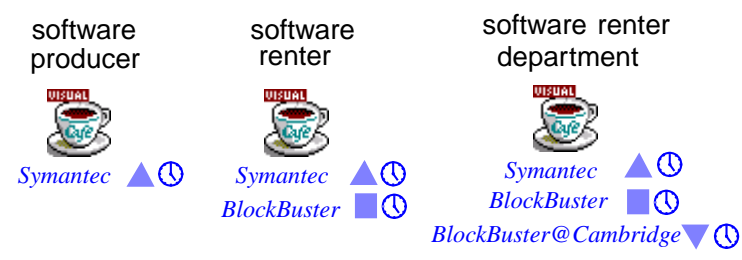

Figure 11. Rent-A-Soft: each level (producer, renter, renting company, and their departments) encapsulates its data and supporting code for maintaining the renting process, upgrades, inventory, etc.

very end, we had found ways around all these problems, but they mostly consisted of ad hoc solutions, and by staying away from ClearCase as much as possible. The bare minimum consisted of maintaining the source code control, and Clearcase was indeed very suitable for this.

At the moment of writing this paper, the MOA system consists of approximately 30,000 lines of code (including comments), organized in 21 packages, 200 classes, and 10 interfaces. This does not include test programs, developed as unit tests for most packages, and 25 test scenarios exercising various aspects of the system. The tests, including the configuration files, represent additional 11,500 lines of code. The footprint (accumulative size of classes) of the whole MOA system, along with the test programs, is approximately $730 \mathrm{~KB}$.

We have only now started working on performance and robustness. We eliminated a few obvious performance bottlenecks and are improving it further. Because of this, and because all measurements were done using interpreted Java (JIT for 1.2 will be available only for the final release on NT platform), results should be taken with the grain of salt. Measurements were conducted between two $100 \mathrm{MHz}$ pentium PCs connected in a separate LAN (10Mb ethernet), running NT 4.0 and JVM 1.2. All measurements are an average of 5 runs, which in turn consist of 1,000 RPCs or of 100 moves, subject to measurement.

The RPC with a null message between two agents running on two different nodes takes approximately $25 \mathrm{~ms}$. Note that even though the context of the message is null, the message itself is not null, since it contains destination and source fields. Serializing this object incurs additional cost. Out of $25 \mathrm{~ms}$, approximately $3 \mathrm{~ms}$ is part of the MOA code before the message is passed to the JVM stream, and it takes $3 \mathrm{~ms}$ since it is read from the JVM stream and until it is delivered to receiving agent. This is when the agent and MOA system are collocated in the same sandbox. If they reside in different sandboxes, it takes $47 \mathrm{~ms}$ (the corresponding times on the write and read path are $11 \mathrm{~ms}$ and $6 \mathrm{~ms}$ ). For comparison, the null RPC using RMI on the same platform was measured at 5 ms.

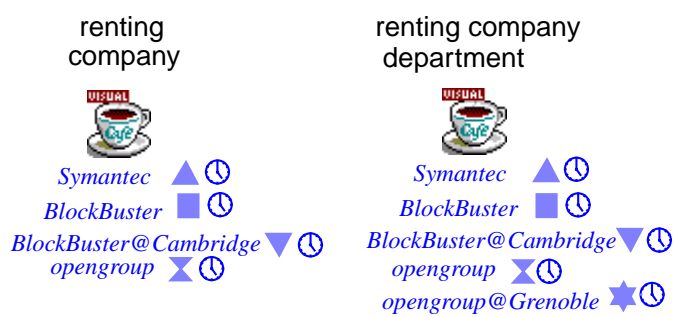

We have measured the move time of a simple agent to be $1177 \mathrm{~ms}$. It takes $88 \mathrm{~ms}$ to serialize the agent and its properties, and $783 \mathrm{~ms}$ to deserialize it. Transfer of the JAR file over network takes approximately $98 \mathrm{~ms}$. For comparison, it takes $45 \mathrm{~ms}$ to transfer the same JAR file using RMI. The higher costs bring in return a higher flexibility, such as forwarding of messages. Nevertheless, we hope that using JIT and further improving the MOA communication will significantly improve the performance.

\section{Applications}

"Rent-A-Soft" is a demo program, presented at Uniforum. The idea is to use agents to help out with distributing and renting software packages. This is applicable for relatively expensive packages, or for cheaper software (such as games) assuming large quantities. A chain of participants is envisioned, such as producer, wholesale renter, renting departments, the company which rents and its own departments which sub-rent and occasionally exchange software. By encapsulating information about the renting source, duration and usage of the package, an otherwise complex inventory tracking process is replaced. Each encapsulated layer can associate an agent responsible for specific activities, such as revoking the rent, statistics maintenance, etc. Communication in the presence of mobility can be used to revoke the rent within the same security domains. For example, the company which rents may revoke certain copies of the package within its own departments, it can install new versions of software, and dynamically monitor software use. The Rent-a-soft application is presented in Figure 11.

Radio Communication. The Grenoble facility of the Open Group Research Institute is planning to use the MOA project for a police force application over radio connections [2]. This is a type of application suited for mobile agents. Radio communication has slow and unreliable links, allowing mobile agents to exploit the locality of reference. Another important feature that prevents alternative solutions based on the client server model to be used as effectively as possible is the unpredictable availability of connections. It can easily happen that connections with the centralized server are broken (e.g. a police car enters a tunnel). In such a case, mobile agents can 
still continue to be functional, e.g. by cooperating with, or visiting other available locations (e.g. another police car that is also in the tunnel).

Security. Even though mobile agents are considered to suffer from (still) inadequate security, they can help to solve some security problems. In certain cases it is not allowed to give access to the actual data stored in a security domain, whereas it is allowed to provide some attributes of the data or some other information about the data. For example, Swiss banks do not allow WWW access to its old accounts since World War II, but they allow anyone checking if the user (or someone from his family, based on the last name) had an account opened. This type of application is a relatively simple query. Imagine a more complex application, where queries need to do complex, arbitrary searches across the whole security domain. Such an application can be easily achieved using mobile agents. A mobile agent would be allowed to enter the security domain. While in the security domain, it can do activities within certain limitations. Before leaving the security domain, agent data could be inspected to see what data it takes out; alternatively only certain data could be allowed to leave. There is an opportunity for establishing covert channels, but depending on how secret the data is and how much information is allowed to leave, this solution could be acceptable in a range of applications. The Open Group RI has an ongoing proposal for the use of mobile agents for improving security.

In all three applications, communication, resource management and interoperability requirements are very important. Our belief is that MOA satisfies these requirements well. For example, communication channels can be temporarily suspended or disabled during the application lifetime, and mobile applications need to reconnect from various sites, requiring the MOA migrateable channel support. For all applications, and especially for security, resource management plays one of the most important roles. Being able to track and limit resources is invaluable for Web server applications. Finally, interoperability is one of the key requirements for many application nowadays, particularly for mobile agents.

\section{Lessons Learned}

In this section we summarize lessons learned while developing MOA.

Operating System Support vs. Middleware. Recently, work in the development of operating systems has significantly reduced. The current trend is toward using NT on lower-end systems and some version of UNIX on highend servers. Linux is a dominating freely available version of an OS, and there are also a few real-time executives. In many cases, operating system modules are being replaced with middleware solutions (true for the MOA project).

Nevertheless, many operating systems techniques can be applied in the development of middleware systems. Again, the same applies in the development of MOA. We have drawn on substantial experience in the area of operating systems, such as communication channels and messaging protocols; locating and naming of mobile agents; resource management; negotiation policies, synchronization among agents, etc.

Transparency in communication (maintaining channels across migration) was more complex to support than we originally thought. We were aware that this is a hard goal to achieve, but we hoped that relaxing assumptions would make it simpler to implement.

Resource management was straightforward to design and implement. We believe that its extensive usage will demonstrate its ultimate benefits even more. We strongly recommend that resource management be initially planned for the development of agent systems. We shall heavily rely on it for some of the future work related to policies for management of agent based systems.

Component-based computing has somewhat slowed us down during the development. Compliance with the component model does not come for free. There are costs both in terms of development effort, as well as run-time. The learning curve was high to get accustomed to Java Beans; we had to provide additional methods to inspect/ set properties; we had to take care that all classes are serializable; we had to create jar files for both the agent application and system; it is required to link (or wire) components once they are loaded. Nevertheless, we feel that the benefits have at least returned the investment so far, and that the benefits will significantly outweigh the investment once we start using and especially configuring the MOA system and MOA applications.

Immediate benefits of complying with the component model were stronger enforcement of component boundaries than is the case with object boundaries. The components are loaded instead of constructed and component boundaries enforced careful design of what is serialized, particularly useful for application development.

In the future, we expect even higher benefits from the component model, allowing for inspection of visiting agents, reconfiguring agent applications, and agent systems. Evolvement of the MOA system will be easier, since changes will be isolated to single components.

Interoperability. It is too early elaborate on the benefits of participating in the OMG MASIF proposal. It was a useful experience to collaborate with implementors of 
other mobile agent systems. We were solving similar problems, sometimes finding different solutions. Because of the different underlying infrastructure, the current compliance is still a future goal, because we need to come up with a reference implementation first. At the moment, we have taken care that nothing stands in the way of the MOA design to prevent us from switching to different communication infrastructure.

\section{Related Work}

There are three classes of related work to MOA. The first class consists of process migration, the second of distributed systems on the Web and the third of mobile agents.

Charlotte process migration [3] dealt with the interprocess communication among the migrating processes and introduced forwarding as a locating scheme. Process migration in the Sprite operating system supported the notion of a home node [12]. In the $\mathbf{V}$ Kernel process migration [31], migrating processes are located by searching them. Emerald supports fine grain mobility on a small scale network, addressing mobility at the language level [19]. In Mach task migration, transparency of communication and resource maintenance is achieved at the microkernel level [23]. A comprehensive survey of process migration is available at [22]. A theoretical description of mobility in form of Actors is presented in [1].

Two distributed object-based systems on the Web explore similar issues as MOA. Legion is an object-based, meta-systems software project, developed at University of Virginia [14] that provides a single, coherent virtual machine and that addresses issues of scalability, fault tolerance, site autonomicity, and security. Globe is an object-based wide-area distributed system constructed as a middleware layer on top of existing networks and operating systems [17]. It is based on the concept of a distributed shared object whose state can be physically distributed and that encapsulates implementation aspects (communication, replication, and migration).

Telescript is the first commercial implementation of the mobile agent concept [34]. Recently, it was discontinued and re-implemented in Java, under the name Odyssey. AgentTcl is a mobile agent system implemented in the Tcl language [20]. It has two components: a special Tcl interpreter that executes the Tcl agents, and a server that runs on each machine to which agents can be sent. It uses the SafeTcl model for security. Aglets is one of the first mobile agent systems written in Java [1]. It supports rich communication semantics (location independency, synchronous, asynchronous and multicast). Mole project at University of Stuttgart was one of the first academic efforts in mobile agents in Java [4]. It collaborates with a few industrial partners, such as Siemens and Tandem. Concordia supports agent persistence and recovery [9]. Collaborative work is based on event manager and two forms of asynchronous distributed events: selected and group-oriented. Ara is a Java-based agent system that applied some changes to the JVM in exchange for increased functionality, such as maintaining thread execution context and imposing limits on memory usage [27]. Tacoma and its descendent $\mathrm{T} 2$ address fault tolerance and security issues [18]. Voyager is a Java-based system for developing distributed applications using mobile objects and agents. It includes an ORB with support for migration, services for persistence, scalable group communication, and basic directory services.

Of the systems presented, the most elaborate schemes for maintaining communication channels across migration were implemented in process migration. This was achieved at the cost of complexity introduced in the operating system [12, 23, 24]. Voyager also supports communication with the migrated away agent, but it relies only on the forwarding strategy. Even though this strategy may appear superior to others (see Section 4.4), it is really the combination of different strategies that offers most benefits to an application writer.

Almost all the systems described provide some support for resource management. None of them, to our knowledge, have made an elaborate use of resource information to pursue negotiation and control.

None of the systems that we described is compliant with the component model. MOA was developed later than most of the agent systems, allowing it to overlap in a timely fashion with the development of Java Beans. This is one of the rare cases when being late happened to be an advantage. Voyager is integrated with the Java Beans event model, but the Voyager system is not built from components.

Of the agent systems we described, Aglets is the only other agent system that plans to pursue a MASIF reference implementation. MOA and Aglets are currently similar with regards to MASIF compliance, i.e. both are Java-, rather than CORBA-oriented. It will be required to adapt security and communication models to adjust to MASIF requirements.

In summary, the MOA system is different from other agent systems in the following unique aspects. The MOA system and applications are Java Beans compliant. The place in MOA can be retained after an agent leaves. Agent naming supports families and generations of agents that can be managed. Agents are tracked using four, per-agent, configurable locating schemes. Communication channels are migrateable. 


\section{Conclusion and Future Work}

In this paper, we described the design and implementation of the MOA project. In particular we presented the MOA object and component models and described its components, such as communication, naming and locating, mobility, and resource management. We also discussed some lessons learned during its development and presented some preliminary performance measurements.

MOA contributions consist of: supporting agent collaboration by maintaining communication channels across migration; providing basic support for denial of service attacks by extensive resource management and negotiation policies; compliance with the Java beans component model, leading to better configurability; and complying with the OMG MASIF standard.

There are many mobile agent systems available nowadays, both from academia and from industry. Even though MOA represents yet another new mobile object system among many research vehicles today, we believe that we have distinguished it sufficiently enough to justify its development. In particular, we believe that it was easier to achieve compliance with the component model while designing the system; similar reasoning applies to managing resources, and to maintaining communication channels.

The lessons we learned range from resemblance of middleware solutions to experience with operating systems. Our experience with the component model distinguishes between costs and benefits of complying with the component model. We strongly believe the latter will outweigh the former already for moderate requirements for configurability. We introduced a lot of complexity by maintaining communication transparency. Resource maintenance proved to be very useful with expectations to significant benefits in the future. Finally, OMG MASIF standard impacted only the design decisions of MOA so far. We expect to learn more about MASIF as we pursue the reference implementations.

The future work consists of four areas. First, we plan to extensively improve security. In particular, we plan to include authentication, authorization, integrity checking, and the trust model of MOA. The second area consists of applications, which we plan to support a few. The third area addresses improvements to the current implementation, in particular related to performance and robustness. Finally, we plan to demonstrate interoperability in practice, by interoperating with another OMG MASIF reference platform, such as Aglets.

\section{Acknowledgments}

We are grateful to Shai Guday and Holger Peine for reviewing this paper. They significantly improved its presentation and contents. Rosemary Hudson and Jackie Clark undertook the impossible task to insert all missing articles and to eliminate the superfluous ones that an author, a non-native English speaker, introduced.

\section{Availability}

The MOA project is available for scientific and research purposes under a Collaborative Research Agreement from The Open Group. The URL of the project is: http:/ /www.camb.opengroup.org/RI/Techno/OS/moa.html.

\section{References}

[1] Agha, G., "A Model of Concurrent Computation in Distributed Systems", MIT Press, Cambridge, MA, 1987.

[2] ANIMA Project, http://www.gr.opengroup.org/anima.

[3] Artsy, Y. and Finkel, R. (September 1989). Designing a Process Migration Facility: The Charlotte Experience. IEEE Computer, pp 47-56.

[4] Baumann, J., Hohl, F., Rothermel, K., Strasser, M., "Mole, Concepts of a Mobile Agent System", to appear in the WWW Journal, Special Issue on Applications and Techniques of Web Agents, Baltzer Science Publishers.

[5] Black, D., Milojicic, D., Langerman, A., Dominijanni, M., Dean, R., Sears, S., "Distributed Memory Management", accepted for publication, Software Practice \& Experience, 1997.

[6] Bradshaw, J., "Software Agents", AAAI/MIT Press, 1996.

[7] Chess, D., Grossof B., Harrison, C., Levine, D., Parris, C., Tsudik, G., "Itinerant Agents for Mobile Computing”, IEEE Personal Communications Magazine, October 1995 .

[8] Cockayne, W., and Zyda, M., "Mobile Agents: Explanations and Examples", Manning, 1997.

[9] Concordia: "Concordia: An Infrastructure for Collaborating Mobile Agents" Proc. of Workshop on Mobile Agents MA'97, Berlin, April 7-8th. LNCS 1219, Springer Verlag.

[10] Cybenko, G., Spontaneous comment during Transportable Agents Workshop, September 1997.

[11] DARPA Broad Agency Announcement, 98-01, "AgentBased Systems", http://ballston.prc.com/baa9801/ abspipv1.htm.

[12] Douglis, F. and Ousterhout, J. (August 1991). Transparent Process Migration: Design Alternatives and the Sprite Implementation. Software-Practice and Experience, 21(8):757-785.

[13] Ford, W., Baum, M., "Secure Electronic Commerce", Prentice Hall, New Jersey, 1997. 
[14] Grimshaw, A., et al. "The Legion Vision of a Worldwide Virtual Computer", CACM, vol 40, no 1, Jan. 1997, pp $39-45$.

[15] Goldszmidt, G., Yemini, Y., "Distributed Management by Delegating Mobile Agents", Proc. of the 15th ICDCS, Vancouver, British Columbia, June 1995.

[16] Guideware Corporation, http://www.guideware.com.

[17] Homburg, P., van Steen, M., and Tanenbaum A., "An Architecture for A Wide Area Distributed System", Proc. of the Seventh SIGOPS European Workshop, Connemara Ireland, September 1986, pp 75-82.

[18] Johansen, D., van Renesse, R., and Schneider, F., "Operating system support for mobile agents", Proc. of the 5th. IEEE HOTOS Workshop, Orcas Island, USA (4th5th May, 1995),.

[19] Jul, E., Levy, H., Hutchinson, N., Black, A., "FineGrained Mobility in the Emerald System", ACM TOCS, vol 6, no 1, February 1988, pp 109-133.

[20] Kotz, D., et al., "Mobile Agents for Mobile Internet Computing", July/August 1997, IEEE Internet Computing, vol 1, no 4, pp 58-67.

[21] Lange, D., Oshima, M., "Java Agent API: Programming and Deploying Aglets with Java", Addison Wesley, expected publication date, Winter 1998. (Aglets Web Page: http://www.ibm.co.jp/trl/projects/aglets/).

[22] Milojicic, D., Douglis, F., Paindaveine, Y., Wheeler, R., Zhou, S., "Process Migration Survey", The Open Group Research Institute, Collected Papers, vol. 5, March 1997.

[23] Milojicic, D., Zint, W., Dangel, A., Giese, P., "Task Migration on the top of the Mach Microkernel", Proc. of the third USENIX Mach Symposium, April 1993, pp 273-290, Santa Fe, New Mexico.

[24] Milojicic, D., Douglis, F., Wheeler, R., Guday, S., "Mobility, and Edited Collection", and "Mobility in Distributed Systems", Addison Wesley, expected dates of publication, Winter 1998 and Fall 1999 respectively.

[25] Odyssey Web Page, http://www.genmagic.com/agents/ odyssey.html.
[26] OMG Mobile Agent Systems Interoperability Facilities Specification (MASIF), OMG TC Document ORBOS/ 97-10-05, also available from http://www.opengroup.org/ $\sim \operatorname{dejan} / \mathrm{maf} / \mathrm{draft} 10$.

[27] Peine, H., and Stolpmann, T., "The Architecture of the Ara Platform for Mobile Agents", Proc of the First Intl Workshop on Mobile Agents MA'97, Berlin, April 7-8, Springer Verlag, http://www.uni-kl.de/AG-Nehmer/Ara

[28] Riggs, R., et al.,"Pickling State in the Java System," Proc. of the USENIX 1996 Conference on Object-Oriented Technologies (COOTS), pp 241-250.

[29] Ranganathan, M., Acharya, A., Sharma, S., Saltz. J.,., "Network-aware Mobile Programs", Proceedings of the Annual Usenix 1997 Conf., January 6-10, Anaheim, California, USA.

[30] Shoch, J., Hupp., J., "The Worms Programs - Early Experience with Distributed Computing", Communications of the ACM, 25 (3), pp 172-180, March 1982.

[31] Theimer, M., Lantz, K., and Cheriton, D. (December 1985). Preemptable Remote Execution Facilities for the V System. Proc. of the 10th ACM SOSP, pp 2-12.

[32] Vitek, J., and Tschudin, C., "Mobile Objects Systems: Towards the Programmable Internet", Springer Verlag, April 1997.

[33] Voyager Technical Overview, ObjectSpace, http:// www.objectspace.com/voyager.

[34] White, J., "Telescript Technology: Mobile Agents", General Magic White Paper (http://www.genmagic.com/ Telescript/Whitepapers/wp4/whitepaper-4.html).

[35] White, J., et al., "System and Method for Distributed Computation Based upon the Movement, Execution, and Interaction of Processes in a Network", United States Patent, no 5603031, February 1997.

[36] Wollrath, A., et al., "A Distributed Object Model for the Java System," Proc. USENIX 1996 Conf. on Object-Oriented Technologies (COOTS), pp. 219-231. 